\title{
Studies of photoconductivity in $\beta$-Apo-8 carotenal
}

\author{
SEEMA SEN and T N MISRA \\ Spectroscopy Department, Indian Association for the Cultivation of Science, Jadavpur, \\ Calcutta 700032, India \\ MS received 26 June 1995

\begin{abstract}
The photoconductive properties of a carotenoid polyene, $\beta$-Apo- $8^{\prime}$ carotenal in polycrystalline form has been studied. The growth of the photocurrent shows an overshoot in proposed to be due to higher value of recombination coefficient than trapping coefficient. From the temperature dependence study it is observed that the steady state photocurrent, at first increases with increase of temperature, attains a maximum at a particular temperature $T_{\max }$ and then decreases with temperature. The $T_{\max }$ value agrees with the temperature above and below which steady state photocurrent is attained differently. Monomolecular and bimolecular recombination processes at two temperature regimes are proposed to account for the observed behaviour. The dependence of photocurrent with excitation light intensity and wavelength study provide information on the carrier generation processes. The fast decay of photocurrent have been observed at different temperatures and from this study the decay constant is calculated and it is found to be temperature independent.
\end{abstract} \\ the growth-time curve before steady state value is attained. This behaviour of photocurrent is
}

Keywords. Polyene; organic photoconductivity; action spectra; growth and decay kinetics.

\section{Introduction}

A photoconductor when exposed to light needs some time to reach the steady state value. The establishment of a steady state photocurrent can become rather complicated owing to the cooperation of different localized traps and recombination centres. It has been observed that under certain conditions the photocurrent reveals anomalous behaviour as it passes through a maximum before the steady state is reached. Further the photocurrent rises up to the maximum and then decays to the steady state value as power function of time with exponents which depends on the trap distribution in the energy space. Though such studies so far has been confined in inorganic semiconductors in general and chalcogenide glasses (Andreish et al 1983; Goel and Kumar 1987; Tripathi and Kumar 1988 ) in particular, little attention has been paid to organic semiconductors which are known as wide band gap materials with various distribution of localized states.

In this paper we report the results of our studies on anomalous rise in photocurrent in a polyene semiconductor.

Highly conjugated structure of polyene molecules results in their semi and photoconducting behaviour in the solid state (Chynoweth and Schneider 1954). In photobiological processes like photosynthesis the photoconduction of polyenes is thought to be involved (Goodwin 1980). The photoconductivity of $\beta$-carotene and its various isomers and of some other polyenes has been studied extensively (Rosenberg 1961, 1966; Hoshino and Tateishi 1979; Pal and Misra 1989). The study of photoconductivity in such materials is interesting since they have a large photocurrent to dark current ratio which is a necessary criteria for photovoltaic effect. Another interesting feature of these samples is that they form charge transfer complex (CT) with iodine and after formation of the complex their conductivity increases by several powers of ten (Jain 


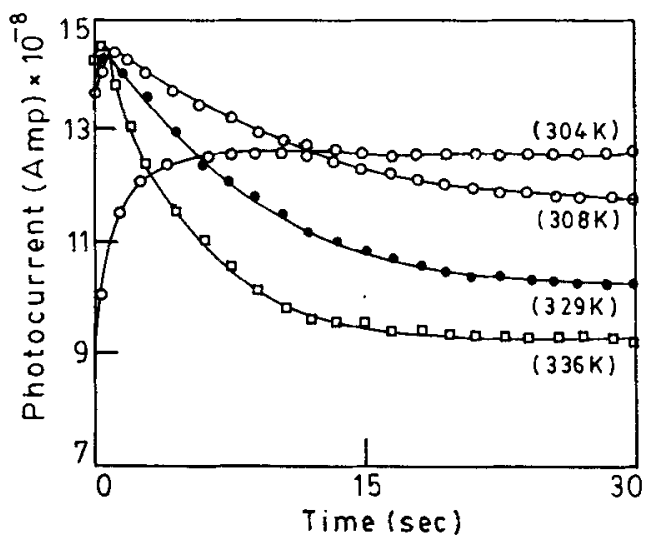

Figure 1. Growth of photocurrent with time at different temperatures at $100 \%$ excitation light intensity at an applied voltage of $30 \mathrm{~V}$.

et al 1988). Moreover the CT complexes of these samples have been used quite successfully as batteries (Jain et al 1988; Sen et al 1993) and in this context this polyene $\beta$-Apo- $8^{\prime}$ carotenal is very promising and the application can be extended to the development of some other devices.

Here we have studied the occurrence of an overshoot in the growth time curve of the photocurrent in the polyene, $\beta$-Apo- $8^{\prime}$ carotenal. The effect of temperature and excitation light intensity on the behaviour of the photocurrent rise and on its steady value have also been studied.

\section{Experimental}

Highly pure $\beta$-Apo- $8^{\prime}$ carotenal used in this investigation was obtained as a gift from M/s Hoffmann-La-Roche Co., Switzerland. The powdered sample was used in a sandwich cell with $\mathrm{SnO}_{2}$ coated semi transparent glass and stainless steel electrode and was kept at a moderate pressure of $4400 \mathrm{~g} / \mathrm{cm}^{2}$ by spring clips. The sandwich cell thickness and area was maintained at $0.005 \mathrm{~cm}$ and $0.25 \mathrm{~cm}^{2}$ respectively. The cell was placed in a suitably designed conductivity chamber (Mallick et al 1979) with a quartz window through which light could pass and illuminate the sample. For current measurements Keithley's programmable electrometer (Model no. 617) was used which has a data storage facility alongwith a built-in isolated d.c. voltage supply which can be adjusted between $-100 \mathrm{~V}$ to $+100 \mathrm{~V}$. A 100 Watt Xenon lamp (XBO 100) was used to illuminate the sample. Various neutral density filters were interposed in the beam path to control the light intensity.

\section{Results and discussion}

\subsection{Anomalous transient photoconductivity}

Generally in any photoconductor the growth of photocurrent is dependent upon excitation light intensity and temperature of the material. Considering this in the 


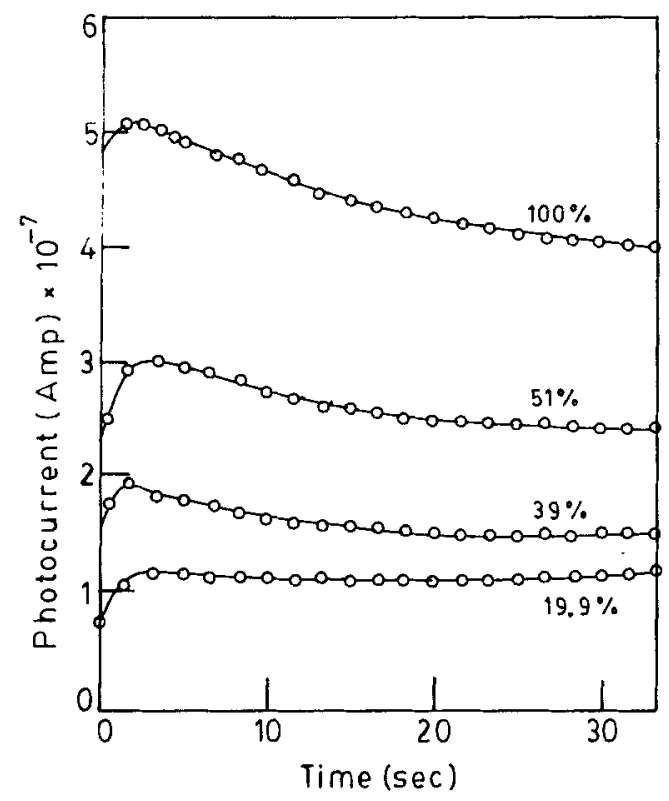

Figure 2. Growth of photocurrent with time at different excitation light intensity at a constant temperature $332^{\circ} \mathrm{K}$ at an applied voltage of $100 \mathrm{~V}$.

present case the growth of photocurrent with time at different temperatures and excitation light intensity were studied and the results are shown in figures 1 and 2 respectively. It is observed that at higher temperature the photocurrent passes through a maximum before attaining a steady state value. This behaviour is generally called anomalous transient photocurrent. Such type of behaviour is rather frequent in case of chalcogenide glasses (Goel and Kumar 1987; Andreish et al 1983; Tripathi and Kumar 1988) but is quite rare for organic substances. In time-temperature regime both $t_{\max }$ (the time needed by the photocurrent $I_{\mathrm{ph}}$, to reach the maximum value from $t=0$ ) and the time $t_{\mathrm{st}}$ (the time needed by $I_{\mathrm{ph}}$ to attain the steady value from the maximum value) decreases rapidly with increase of temperature. It is also clear from figure 1 that there is a temperature $\left(T_{\max }\right)$ above and below which the steady state photocurrent is attained differently. The significance of this temperature $T_{\max }$ is that at $T=T_{\max }$, value of $I_{\mathrm{st}}^{\mathrm{ph}}$ is highest and below and above $T_{\max }, I_{\mathrm{st}}^{\mathrm{ph}}$ falls off (figure 4).

At $T>T_{\max }$ the photocurrent passes through a maximum before attaining the steady state value whereas at $T<T_{\max }$ the photocurrent rises slowly to attain the steady state value without passing through an overshoot. However, since growth is intensity dependent, at very high intensity, at $T>T_{\max }$ also there may be the occurrence of an overshoot. According to the model proposed by Kastner and Monroe (1982) and Andreish et al (1983) this phenomenon occurs at high photocurrent generation rate due to non-equilibrium distribution of recombination centres and predominance of bimolecular recombination. When the recombination coefficient $\left(b_{\mathrm{r}}\right) \gg b_{\mathrm{t}}$, the trapping coefficient, then there will be such an overshoot of photocurrent before the attainment of the steady state. 


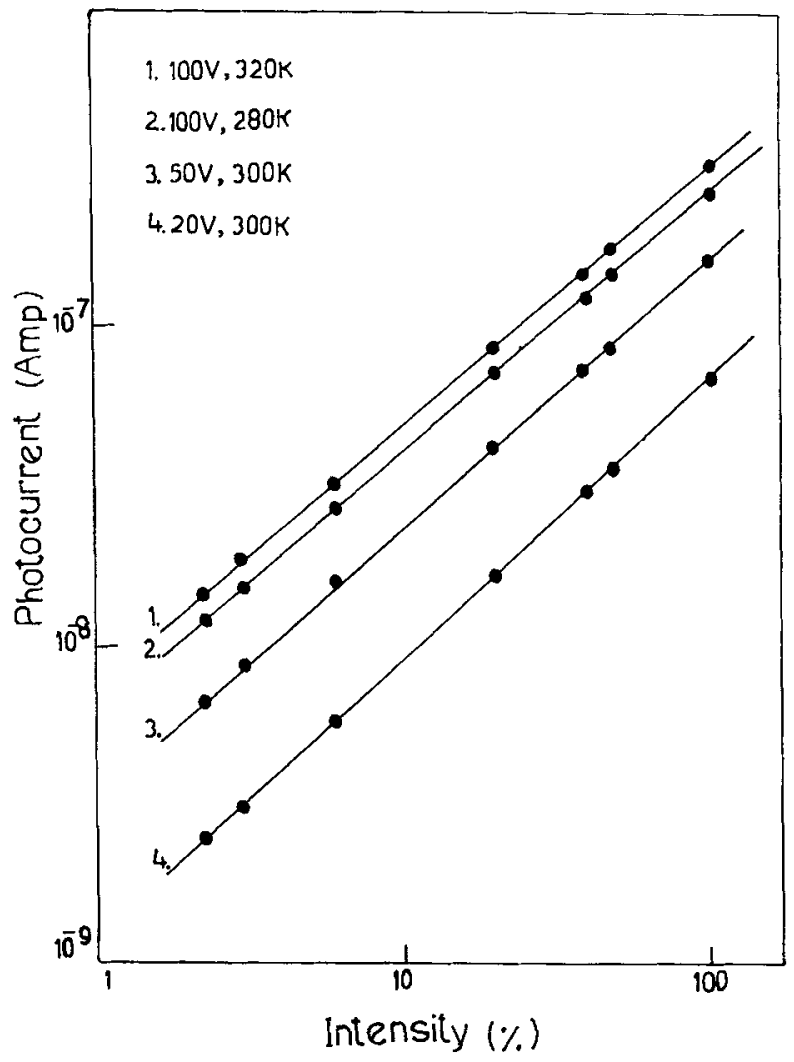

Figure 3. Steady state photocurrent vs light intensity dependence in logarithmic scale at different applied voltages and temperatures.

\subsection{Excitation light intensity dependence of photocurrent}

The excitation light intensity dependence of steady state photocurrent $I_{\mathrm{ph}}^{\mathrm{st}}$ have been studied and is shown in figure 3 . It is found that over the entire range of working intensity the photocurrent $I_{\mathrm{ph}}$ is related with the excitation light intensity $I_{\mathrm{B}}$ through the power law

$$
I_{\mathrm{ph}} \propto I_{\mathrm{B}}^{\gamma}
$$

For the whole range of excitation intensity the value of $\gamma$ is obtained as 0.85 and this behaviour remains voltage and temperature independent throughout the whole range of intensity.

The sublinear behaviour of photocurrent on light intensity has been thought (Rose 1963) to be due to cooperation between a set of recombination centres with traps. In case of large electron trapping cross-section the equilibrium concentration of holes present under illumination exceeds electron concentration and $p$-type conductivity is observed. Also the quasi Fermi level $E_{\mathrm{f}(p)}$ is shifted towards valence band on illumination and trapping centres now act as recombination centres. This results in sublinear behaviour of $I_{\mathrm{ph}}$ on light intensity and $\gamma$ lies between 0.5 and 1 . 


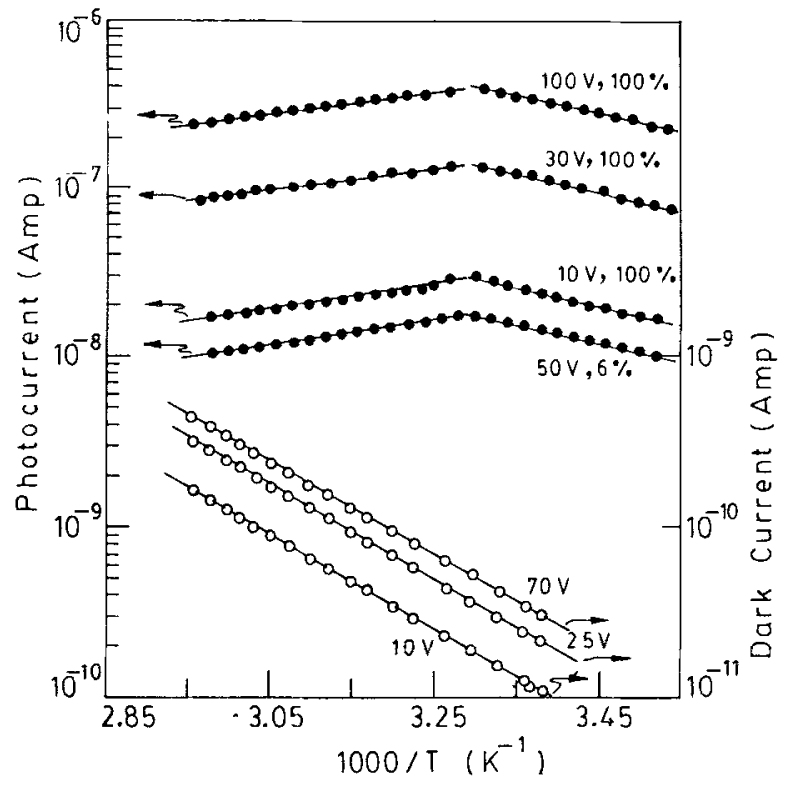

Figure 4. The plots of $\log I$ vs $1000 / T$ at various applied voltages with illuminated electrode + ve. $\left(-\mathrm{O}^{-} \mathrm{O}^{-}\right)$represents dark and (--) represents photoconductivity with polychromatic light at two different intensities.

\subsection{Temperature dependence of steady state photocurrent}

The dependence of dark and steady state photoconductivity on temperature at various applied fields at two different excitation light intensities have been studied (figure 4). It is clear from the figure that although the dark current shows a typical feature of a semiconductor i.e. semilogarithmic plots of dark current vs 1000/ $T$ yields a straight line from which the value of dark activation energy is obtained as $1 \cdot 1 \mathrm{eV}$; the photocurrent shows some unusual behaviour. With increase in temperature the photocurrent increases showing the usual behaviour of photoconductor and satisfies the relation

$$
I_{\mathrm{ph}}=A \exp \left(-\Delta E_{\mathrm{ph}} / K T\right)
$$

up to a particular temperature (say) $T_{\max }$. After $T_{\max }, I_{\mathrm{ph}}^{\mathrm{st}}$ decreases with increase of temperature. Similar observation of maximum photoconductivity at a specific temperature has been found in chalcogenide glasses (Anderson and Spear 1977; Tripathi and Kumar 1988), in amorphous Si (Spear et al 1979) and in a biomaterial crocetene (Pal and Misra 1989). In chalcogenide glasses this has been shown to be due to different recombination processes in the two temperature regimes; the bimolecular recombination at high temperature regime and monomolecular recombination at low temperature regime.

We believe that in the present case also predominance of different recombination processes in the two temperature regimes is responsible for this unusual behaviour. The photoconductivity is given by (Meier 1974)

$$
\sigma_{\mathrm{ph}}=e \mu\left(\eta I_{\mathrm{B}} / k_{\mathrm{r}}\right)^{1 / r}
$$


where $\mu$ the mobility, $\eta$ the quantum yield of carrier generation and $k_{\mathrm{r}}$ (the recombination rate) are the established temperature dependent quantities. The activation energy is to be derived from the temperature dependence of one or more of these quantities. Here $r=1$ is for monomolecular recombination whereas $r=2$ is for bimolecular recombination. Observed temperature dependence of photoconduction above $T_{\max }$ may indicate exponential dependence of bimolecular recombination rate on temperature.

However, our light intensity dependence of photoconduction does not show any significant temperature dependence. The recombination pattern is governed not only by excitation light intensity and temperature but by the total number of carriers present (both dark and photo). In the present case with increase of temperature dark current $\left(I_{\mathrm{d}}\right)$ increases much more rapidly than photocurrent $\left(I_{\mathrm{ph}}\right)$ and after $T_{\max }, I_{\mathrm{d}}$ increases with increase of temperature but $I_{\mathrm{ph}}$ decreases and as a result with increase of temperature dark and photo carrier concentration becomes approximately comparable. This may be the cause of the observed recombination pattern in the intensity dependence. Alternatively, recombination of excess electrons into the hole distribution which is only slightly disturbed by illumination has been proposed to cause such photoconducting behaviour in amorphous silicon (Spear et al 1979). Scattering of excitons by phonons whose density of states increases at high temperatures or activated exciton trapping in deep traps, may also reduce photoconductivity at high temperature.

\subsection{Action spectra}

By studying the photoconduction action spectra one can determine the carrier generation processes. Usually for most organic substances the spectral response of $I_{\mathrm{ph}}$ corresponds to the absorption spectra (Chaiken and Kearns 1966; Meier and Albrecht 1969; Lyons and Newmann 1971) i.e. symbatic action spectra is produced. This similarity suggests that the primary step leading to carrier generation is excitation of levels which corresponds to absorption spectra. But in some organic substances the minima of absorption spectra coincides with maxima in photoconduction spectra i.e. an antibatic action spectrum is produced. It is found that for the case of production of

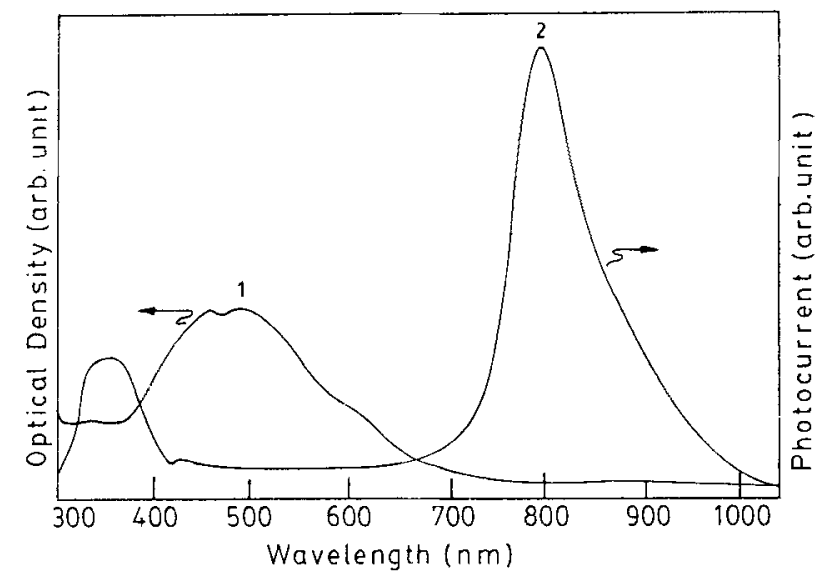

Figure 5. The wavelength dependence of photocurrent and absorption spectrum in solid film: (1) for absorption spectrum of thin film and (2) for photoconduction action spectrum with illuminated electrode + ve. 
antubatic spectrum there should be a shift in the action spectrum with the change of polarity of the illuminated electrode (Goddewski and Kalinowski 1979).

As is shown in figure 5 , in $\beta$-A po- 8 ' carotenal, photoconduction action spectra show one short wavelength band in the region $300-400 \mathrm{~nm}$ and one long wavelength band in the $700-1050 \mathrm{~nm}$ region but the absorption spectrum of this material contains only one prominent band in the region $400-600 \mathrm{~nm}$. However the voltage dependence of the action spectrum does not show any shift in the band position from which we may conclude that this is not an antibatic spectrum (Goddewski and Kalinowski 1979). The short wavelength peak in the action spectrum of $\beta$-carotene has been attributed to photoinjection of charge carriers from the illuminated electrode (Hoshino and Tateishi 1979). If photoinjection of charge carriers from the electrodes is responsible, then the Fowler relation (Williams and Dresner 1967) should be applicable and the photocurrent quantum yield $Q$ should satisfy the relation

$$
Q \propto\left(\Phi-\Phi_{i}\right)^{2}
$$

where $\Phi$ is the photon energy and $\Phi_{i}$ the electron/hole photoinjection threshold energy. A plot of square root of photocurrent vs the photon energies should be linear and from the plot photoinjection threshold energy should be obtainable. Since here the structure of photoaction spectra is same for both positive and negative illuminated electrode, both the electron photoinjection threshold energy and the hole photoinjection threshold energy should be same as was found in case of anthracene (Many et al 1967). Such a Fowler plot is shown in figure 6, from which the photoinjection threshold energy is obtained as $2.73 \mathrm{eV}$. This is quite similar to the value obtained by Hoshino and Tateishi (1979) in another polyene material.

The feature of the long wavelength band is distinctly different from the absorption spectrum. Such long wavelength peak has been reported for many other polyene materials (Rosenberg 1966; Chapmann et al 1967; Hoshino and Tateishi 1979; Pal and

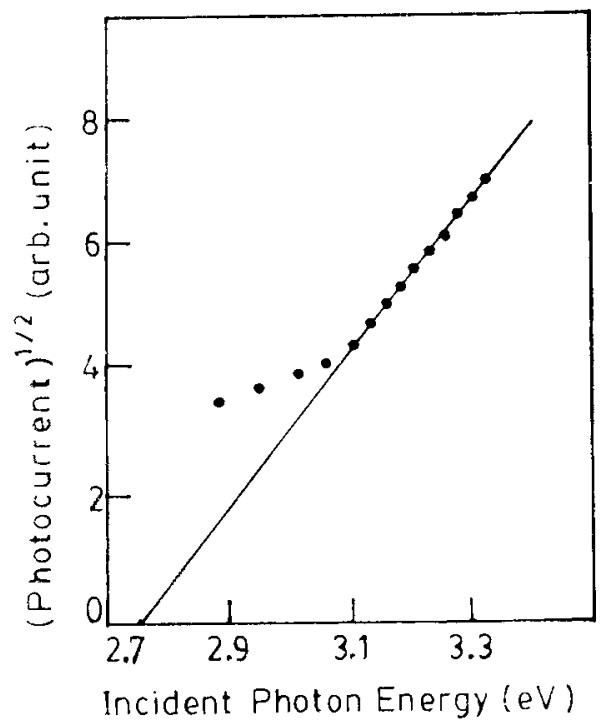

Figure o. Fowler plot for $\mathrm{SnO}_{2}$ electrode maierial. 
Misra 1989). The long wavelength tail of this photoconduction band when extrapolated, intersect the abscissa at an energy which gives the photoconduction threshold energy i.e. the photon energy at which the compound displays photoconductivity. For this material the value of this threshold energy comes about $1.25 \mathrm{eV}$, whereas the value of dark activation energy $E_{\mathrm{d}}$ is obtained as $1 \cdot 1 \mathrm{eV}$ (figure 4). This close agreement suggests that the intrinsic mechanism of carrier generation (direct electron-hole production) is operative both in dark and photoconduction.

\section{Acknowledgement}

It is our great pleasure to thank M/s Hoffmann, La Roche Co. Ltd., Switzerland for generous gift of the polyene material.

\section{References}

Anderson D A and Spear W E 1977 Philos. Mag. 36696

Andreish A M, Arkhipov V I, Iovn M S, Rudenko A I and Shutov S D 1983 Solid State Commun. 481041

Chaiken R F and Kearns D R 1966 J. Chem. Phys. 453966

Chapman D, Cherry R J and Morrison A 1967 Proc. R. Soc. A301 173

Chynoweth A G and Schneider W G 1954 J. Chem. Phys. 221021

Goddewski J and Kalinowski J 1979 Phys. Status Solidi (c) 56 k57

Goel S and Kumar A 1987 Solid State Commun. 64371

Goodwin T W 1980 The carotenoids (London: Chapman and Hall) 2nd Ed., Vol. II

Hoshino Y and Tateishi K 1979 J. Phys. Soc. Jpn. 4672

Jain K M, Abraham S and Hundet A 1988 Jpn. J. Appl. Phys. 27867

Kastner M A and Monroe D 1982 Solid Energy Mater. 841

Lyons L E and Newmann O M G 1971 Aust. J. Chem. 2413

Mallick B, Ghosh A and Misra T N 1979 Bull. Chem. Soc. Jpn. 522091

Many A, Levinson J and Teucher I 1967 U.S. Clearinghouse Fed. Sci. Techn. Inform. AD 822571

Meier H 1974 Organic semiconductors: dark and photoconductivity of organic solids (Germany: Verlag Chemie)

Meier H and Albrecht W 1969 Ber. Bunsenges. Physik. Chem. 7386

Pal P and Misra T N 1989 J. Phys. D: Appl. Phys. 221358

Rose A 1963 Concepts in photoconductivity and allied problems (USA: Interscience)

Rosenberg B 1961 J. Opt. Soc. Am. 51238

Rosenberg B 1966 Advances in radiation biology (New York: Academic Press) Vol. II, p. 193

Sen S, Pal P and Misra T N 1993 J. Mater. Sci. 281367

Spear W E, Loveland R J and Sharabati A L A 1979 J. Non-Cryst. Solids 15410

Tripathi S K and Kumar A 1988 Thin Solid Films 16599

Williams R and Dresner J 1967 J. Chem. Phys. 462133 